\title{
Accuracy of the chest $x$-ray in screening for tuberculosis in Uganda: A cross-sectional study.
}

Joanitah Nalunjogi ( $\nabla$ jmukwaba@gmail.com )

Makerere University College of Health Sciences https://orcid.org/0000-0002-9574-6846

Frank Mugabe

Makerere University Lung Institute

Irene Najjingo

Makerere university Lung Institute

Pastan Lusiba

Makerere University College of Health Sciences

Francis Olweny

Makerere University College of Health Sciences

Joseph Mubiru

Makerere University Lung Institute

\section{Edward Kayongo}

Makerere University College of Health Sciences

\section{Rogers Sekibira}

Makerere University Lung Institute

\section{Achilles Katamba}

Makerere University Lung Institute

\section{Bruce Kirenga}

Union Internationale contre la Tuberculose et les Maladies Respiratoires

\section{Research}

Keywords: CXR, symptoms, accuracy, screening, Tuberculosis, chest radiograph, TB, Uganda

Posted Date: June 26th, 2020

DOI: https://doi.org/10.21203/rs.3.rs-37900/v1

License: (c) (i) This work is licensed under a Creative Commons Attribution 4.0 International License. Read Full License 


\section{Abstract}

ABSTRACT BACKGROUND: The WHO END TB strategy requires $\geq 90 \%$ case detection to combat tuberculosis (TB). Increased TB case detection requires a more sensitive and specific screening tool. Currently, the symptoms recommended for screening TB have been found to be sub-optimal. CXR as a screening tool for pulmonary TB was evaluated in this study, as well as factors related to its false positive results. METHODS: A cross sectional study of 4441 records of consented/assented participants $\geq 15$ years. Participants with a cough $\geq 2$ weeks and/or any abnormality in the lung on CXR were included in the study. Löwenstein-Jensen $(\mathrm{LJ})$ culture was used as the gold standard. The CXR were categorised as Abnormal meaning presence of any CXR abnormality suggestive of active tuberculosis. Symptoms were categorised as abnormal meaning presence of any of cough $\geq 2$ weeks, fever, weightloss or night sweats. RESULTS: The CXR had sensitivity $93 \%$, specificity $65 \%$ compared to culture results while symptoms had sensitivity $76 \%$ and specificity $31 \%$. The adjusted prevalence ratio (APR) of a false positive CXR result increased with age categories (years); 45 - 54, APR 1.18 (1.08, 1.29), 55 - 64 APR 1.18 $(1.09,1.29), 65$ +years APR $1.2(1.10,1.30)$. The APR was $0.93(0.90,0.96)$ among males and $0.86(0.79,0.93)$ among HIV positive individuals. CONCLUSIONS: The CXR is a fair tuberculosis screening tool and performed better than symptoms in Uganda.

\section{Background}

The WHO 2018 estimates show that Tuberculosis (TB) was responsible for 1.2 million deaths among HIV negative persons and 250,000 deaths among HIV positive persons. ${ }^{(1)}$ Uganda has a high TB incidence of 201 per 100,000 population as well as high TB related mortality of 26 per 100,000 population. The TB case detection rate for Uganda is $72 \% .{ }^{(2)}$

In order to combat tuberculosis, WHO has proposed three strategies; intensified case finding (ICF), isoniazid preventive therapy (IPT) and infection control (IC). ${ }^{(3)}$ The WHO END TB strategy indicates a need for $\geq 90 \%$ TB case detection among others to combat TB. ${ }^{(4)}$

The strategic plan for the Uganda national tuberculosis and leprosy programme (NTLP) seeks to achieve $85 \%$ case detection by 2019/2020. However, confirming TB is difficult. Currently TB culture is the gold standard but financial and logistic challenges make it difficult to scale up. There is need to explore more widely available, low cost screening and diagnostic tools and algorithms to aid TB diagnosis. The CXR is one such potential tool.

The currently employed symptoms screening tool for tuberculosis in Uganda has been observed to have sensitivity $40.7 \%$ and specificity of $81.3 \%$ according to a survey done in South Africa considering presence of any cough, fever, weightloss or Night sweats. ${ }^{(5)}$ Systematic reviews done to assess the performance of the symptoms in high HIV prevalence regions have shown sensitivity $84 \%$ and specificity $74 \% .{ }^{(6)}$ The symptoms screening tool runs a risk of missing patients with tuberculosis as the patients do not seek care and do not have symptoms due to early TB disease.

The chest radiograph (CXR) was commonly used in mass screening campaigns in Europe and the US in the early TB chemotherapy era (1950s), but has not been widely used in screening TB in low-income countries because of high cost and lack of capacity. $(7,8)$ This CXR has been found to have sensitivity of $98 \%$ and specificity of $75 \%$ according to a systematic review. ${ }^{(6)}$

The recognition that bold new strategies are needed to control TB (END TB Strategy) has led to reconsideration of CXR as a potential tool, as innovative, lower costs strategies for performing CXR (digital radiography) and reading them (computer aided reading) have become available. ${ }^{(9,10)}$ The use of more sensitive tuberculosis screening tools increases the pool of presumptive TB cases hence increasing case detection as more individuals are exposed to the confirmatory test which ultimately leads to reduction in mortality and morbidity. ${ }^{(11)}$

The impact of false positive results in screening of tuberculosis.

Page 2/15 
The performance of screening tests is affected by the level of false negative and false positive results that are obtained as a result of using the test. The CXR and symptoms have been reported to have a very high number of false positive results hence challenges in their application as many individuals who would have been saved from a confirmatory test will still undergo the confirmatory test only to find them without the disease. ${ }^{(12,13)}$

The identification of the factors responsible for false positive results is important as it guides the application of the test to individuals who are more likely to benefit from the test. A study done in Tennessee found a high rate of normal CXR among PLHIV with culture confirmed tuberculosis. ${ }^{(14)}$ These factors if identified help guide the implementation of screening interventions in the various populations and countries.

Uganda carried out a national tuberculosis prevalence survey (UTPS) from 2014-2015 which screened for tuberculosis by collecting data on TB symptoms using questionnaires and performed CXR in all consenting participants. ${ }^{(15)}$

Therefore using the data from UTPS, we sought to evaluate the performance of the Chest radiograph in the screening of tuberculosis and factors associated with a false positive CXR.

\section{Methods}

\section{Study design}

We conducted a cross sectional study by secondary analysis of the data collected during the Uganda National Tuberculosis prevalence survey (UTPS).

\section{Study design, setting and sampling method used in the UTPS}

The Uganda National TB prevalence survey was conducted from October 2014 to July 2015 with the primary objective of determining the prevalence of Tuberculosis in Uganda. Villages (clusters) across the country were sampled using probability proportionate to size (PPS). The starting block was randomly selected by the village leader and then blocks were added in a clockwise manner around the original block until the required cluster size was achieved. Eligible respondents aged $\geq 15$ years in selected blocks were invited to the survey ${ }^{(15)}$.

\section{Study procedures Screening strategy}

All eligible consenting/assenting individuals who were $\geq 15$ years were screened for tuberculosis by research assistants in the community using a questionnaire which listed the symptoms and a CXR (Fig. 1). Parents/Guardians of individuals < 18 years also offered informed consent.

Participants with a cough $\geq 2$ weeks and/or any abnormality in the lung on CXR were considered presumptive for TB and were requested to submit two sputum samples (a spot and an early morning sample).

Respondents who did not have a CXR taken were also eligible to submit sputa, even if asymptomatic.

All respondents eligible for sputum collection had HIV testing.

\section{CXR reading}

CXRs were read in the field by a trained technician and interpreted as normal or abnormal. Two experienced independent radiologists categorised all the abnormal CXRs as normal, suggestive of active TB disease, inactive/healed TB, and extra pulmonary abnormalities. A third radiologist adjudicated in case there were differences and the differences were resolved by consensus. All normal x-rays were read a single time. 


\section{The Gold standard}

The culture results obtained with Löwenstein-Jensen medium and confirmed as survey TB were considered as the gold standard.

\section{Study participants}

Records of participants $\geq 15$ years were included in the study. We excluded records that were missing data on culture and CXR results.

\section{Sampling and sample size}

All records in the electronic database that met the inclusion criteria for the study were analysed.

To estimate the sample size for performance of the CXR and symptoms. The modified Kish Leslie formula for diagnostic studies was used. We assumed $95 \%$ confidence level $(\mathrm{Cl})$, sensitivity $90 \%$, specificity $83 \%{ }^{(16)}$ and TB prevalence of 401 per 100,000 population ${ }^{(15)}$ to obtain the desired sample size for assessing the performance of the CXR in screening for tuberculosis.

\section{Data extraction}

We used a data extraction form to obtain variables of interest from the electronic database.

The CXR results were considered as abnormal (suggestive of active TB disease) or Normal (healed TB/inactive TB, other TB related lung abnormalities, other non-TB related lung abnormalities and extra pulmonary abnormalities, normal).

The symptoms screen encompassed presence of cough of $\geq 2$ weeks, fever, weight loss and night sweats as recommended by Uganda Ministry of Health. Individuals with any of the above symptoms were considered presumptive for tuberculosis.

\section{Data analysis}

We used the STARD 2015 guidelines in reporting the results of this study ${ }^{(17)}$. Data was analysed using Stata version 13.0 (College Station, Texas, USA). In our study, records with any of the above symptoms i.e. cough $\geq 2$ weeks, fever, weightloss or night sweats were analysed as positive for symptoms. Records indicating CXR abnormalities suggestive of active TB were analysed as positive by the CXR.

Variables were summarised using percentages and proportions depending on their distribution. Performance of the symptoms screening and CXR were reported as sensitivity, specificity, negative and positive predictive value, negative and positive likelihood ratios.

The chi square was used to determine the association between each predictor and false positive CXR results. These were reported as prevalence ratios due to the high prevalence of both CXR and symptoms false positive results in the population.

The Poisson multivariate regression model with robust standard errors was used to obtain factors that were independently associated with false positive CXR or symptoms. The results were reported as prevalence ratios because the prevalence of false positive results was greater than $10 \%$. All factors with level of significance $\leq 5 \%$ were considered significant. Interaction was assessed using the chunk test and considered present at P-value $<0.05$. Confounding was assessed and considered present at $\geq 10 \%$ difference in the prevalence ratio.

\section{Results}

Study Profile 
A total of 40,539 participants were screened for TB in the UTPS. We excluded 35,690 records of participants that did not submit sputum to the laboratory. We excluded 348 records because they were missing data on culture results. 60 records were excluded due to missing data on CXR results. The remaining 4441 records were analysed (Fig. 2).

Characteristics of the study population

The study included individuals aged 15-65 years. Majority of the participants were between ages 25-34 years 832(19\%) and 35-44 years 851 (19\%). There was an equal number of females 2222(50\%) and males (Table 1).

Table 1

Demographic, clinical and radiographic characteristics of the study population

\begin{tabular}{|ll|}
\hline Characteristic & $\mathbf{N}=\mathbf{4 4 4 1} \mathbf{N}(\%)$ \\
\hline Age & \\
25-24yrs & $753(17)$ \\
35-44yrs & $832(19)$ \\
45-54yrs & $851(19)$ \\
55-64yrs & $717(16)$ \\
65+yrs & $468(11)$ \\
\hline Female & $820(18)$ \\
\hline Education & $2222(50)$ \\
None & \\
Primary & $1364(29)$ \\
Secondary & $2247(47)$ \\
Tertiary & $939(20)$ \\
\hline Employed & $212(4)$ \\
\hline Rural residence & $3160(71)$ \\
\hline Smoking Status & $2854(64)$ \\
\hline Never & $3216(72)$ \\
Current & $643(15)$ \\
Past & $582(13)$ \\
\hline HIV & $4957(37)$ \\
\hline Positive & \\
\hline Negative & \\
\hline
\end{tabular}

Performance of the Chest radiograph or symptoms in screening tuberculosis in Uganda Pulmonary tuberculosis was confirmed in 160 (3.6\%) participants using LJ culture. The CXR had sensitivity 93\% (95\%Cl; 87, 96), Specificity $65 \%(95 \% \mathrm{Cl} ; 63,66)$ compared to symptoms with sensitivity $56(95 \% \mathrm{Cl} ; 48,64)$ and specificity $41 \%(95 \% \mathrm{Cl} ; 40$, 43). 
The negative and positive predictive values of the CXR was $99 \% \& 9 \%$ versus $96 \% \& 3 \%$ of the symptoms. The positive and negative likelihood ratios of the CXR were $2.6(95 \% \mathrm{Cl} ; 2.5,2.8), 0.1(95 \% \mathrm{Cl} ; 0.1,0.2)$ compared to symptoms with positive and negative likelihood ratios of $0.9(95 \% \mathrm{Cl} ; 0.8,1.1), 1.1(95 \% \mathrm{Cl} 0.9,1.3)$ (Table 2$)$.

Table 2

Performance of the CXR or symptoms in screening culture confirmed pulmonary tuberculosis.

\begin{tabular}{|lllllllll|}
\hline Diagnostic/ & $\begin{array}{l}\text { Culture } \\
\text { confirmed } \\
\text { TB }\end{array}$ & $\begin{array}{l}\text { Total } \\
\text { Number }\end{array}$ & $\begin{array}{l}\text { Sensitivity } \\
(95 \% \mathrm{Cl})\end{array}$ & $\begin{array}{l}\text { Specificity } \\
(95 \% \mathrm{Cl})\end{array}$ & $\begin{array}{l}\text { Positive } \\
\text { Predictive } \\
\text { Value } \\
(95 \% \mathrm{Cl})\end{array}$ & $\begin{array}{l}\text { Negative } \\
\text { Predictive } \\
\text { Value } \\
(\mathbf{9 5 \% C l})\end{array}$ & $\begin{array}{l}\text { Positive } \\
\text { Likelihood } \\
\text { ratio }(95 \% \\
\text { Cl) }\end{array}$ & $\begin{array}{l}\text { Negative } \\
\text { Likelihood } \\
\text { ratio }(95 \% \\
\text { Cl) }\end{array}$ \\
\hline Prevalence & 160 & 4441 & & & & & & \\
\hline CXR & 148 & 4441 & $93(87,96)$ & $65(63,66)$ & $9(8,10)$ & $99(99,100)$ & $2.6(2.5,2.8)$ & $0.1(0.1,0.2)$ \\
\hline Symptoms & 89 & 4441 & $56(48,64)$ & $41(40,43)$ & $3(3,4)$ & $96(95,97)$ & $0.9(0.8,1.1)$ & $1.1(0.1,0.2)$ \\
\hline
\end{tabular}

Additional Tuberculosis cases obtained by screening with the CXR in addition to symptoms

A total of 160 cases of tuberculosis were diagnosed during the Uganda tuberculosis prevalence survey. Using the CXR in screening tuberculosis an additional $38(23.8 \%)$ cases of tuberculosis were diagnosed. The results are shown in Fig. 3, below.

Factors associated with false positive results on screening with the CXR or symptoms.

A total of 1657 (37\%) of all the participants had abnormal CXR results. Out of all participants with abnormal CXR results, $9 \%$ $(148 / 1657)$ were confirmed to have tuberculosis.

On bivariate analysis, factors associated with a false positive CXR results included; age 45-54 yrs. PR $1.14(1.04,1.24), 55-$ 64 years PR $1.16(1.07,1.27), 65$ + years PR $1.2(1.11,1.30)$ and current smoking PR $1.08(1.06,1.10)($ Table 3$)$. 
Table 3

Bivariate analysis of the factors associated with a false positive CXR result

\begin{tabular}{|c|c|c|c|c|c|}
\hline Characteristic & $\begin{array}{l}\text { True } \\
\text { positive } \\
\mathbf{N}(\%)\end{array}$ & $\begin{array}{l}\text { False } \\
\text { Positive } \\
\text { N (\%) }\end{array}$ & $\begin{array}{l}\text { Prevalence } \\
\text { Ratio }\end{array}$ & $95 \% \mathrm{Cl}$ & P-value \\
\hline \multicolumn{6}{|l|}{ Age } \\
\hline $15-24 y r s$ & $27(19)$ & 116(81) & 1.00 & & \\
\hline $25-34 y r s$ & $37(16)$ & 191(84) & 1.03 & $0.94-1.14$ & 0.518 \\
\hline $35-44 y r s$ & $38(13)$ & $265(87)$ & 1.08 & $0.99-1.18$ & 0.101 \\
\hline $45-54 y r s$ & $23(7)$ & 288(93) & 1.14 & $1.04-1.24$ & 0.002 \\
\hline $55-64 y r s$ & $11(6)$ & $187(94)$ & 1.16 & $1.07-1.27$ & 0.001 \\
\hline $65+y r s$ & $12(3)$ & 462(97) & 1.20 & $1.11-1.30$ & $<0.001$ \\
\hline \multicolumn{6}{|l|}{ Sex } \\
\hline Female & $35(5)$ & $661(95)$ & 1.00 & & \\
\hline Male & 113(12) & $848(88)$ & 0.93 & $0.91-0.96$ & $<0.001$ \\
\hline \multicolumn{6}{|l|}{$\begin{array}{l}\text { Employment } \\
\text { status }\end{array}$} \\
\hline \multicolumn{6}{|l|}{ Unemployed } \\
\hline Employed & $51(10)$ & $458(90)$ & 0.98 & $0.95-1.02$ & 0.317 \\
\hline \multicolumn{6}{|l|}{ Setting } \\
\hline Rural & $73(8)$ & $901(92)$ & 1.00 & & \\
\hline Urban & $75(11)$ & 608(89) & 0.96 & $0.93-0.99$ & 0.018 \\
\hline \multicolumn{5}{|l|}{$\begin{array}{l}\text { Smoking } \\
\text { status }\end{array}$} & \\
\hline & $36(13)$ & $240(87)$ & 1.08 & $1.06-1.10$ & $<0.001$ \\
\hline $\begin{array}{l}\text { Current } \\
\text { Past }\end{array}$ & $32(10)$ & $288(90)$ & 0.97 & $0.93-1.01$ & 0.191 \\
\hline \multicolumn{6}{|l|}{ HIV } \\
\hline Negative & $96(8)$ & $1179(92)$ & 1.00 & & \\
\hline Positive & $37(22)$ & 133(78) & 0.85 & $0.78-0.92$ & $<0.001$ \\
\hline
\end{tabular}

On multivariate analysis the factors found to be independently associated with a false positive result included age categories 35-44 year APR 1.11 (1.01, 1.22), 45-54 years APR 1.18 (1.09, 1.29), 55-64 years APR 1.18 (1.09, 1.29), $65+$ years APR 1.2 $(1.10,1.30)$ while male sex APR $0.93(0.90,0.96)$, positive HIV status APR $0.86(0.79,0.93)$ reduced the false positive CXR outcome (Table 4). 
Table 4

Multivariate analysis of the factors associated with a false positive CXR result

\begin{tabular}{|c|c|c|c|}
\hline Characteristic & $\begin{array}{l}\text { Adjusted prevalence } \\
\text { Ratio (APR) }\end{array}$ & $95 \% \mathrm{Cl}$ & P-value \\
\hline \multicolumn{4}{|l|}{ Age } \\
\hline $15-24 y r s$ & 1.00 & & \\
\hline $25-34 y r s$ & 1.05 & \multirow{2}{*}{$\begin{array}{l}0.95- \\
1.16\end{array}$} & 0.369 \\
\hline $35-44 y r s$ & 1.11 & & 0.028 \\
\hline $45-54 y r s$ & 1.18 & $\begin{array}{l}1.01- \\
1.22\end{array}$ & $<0.001$ \\
\hline $55-64 y r s$ & 1.18 & $\begin{array}{l}1.08- \\
1.29\end{array}$ & $<0.001$ \\
\hline \multirow[t]{2}{*}{$65+y r s$} & \multirow[t]{2}{*}{1.20} & $\begin{array}{l}1.09- \\
1.29\end{array}$ & \multirow[t]{2}{*}{$<0.001$} \\
\hline & & $\begin{array}{l}1.10- \\
1.30\end{array}$ & \\
\hline \multicolumn{4}{|l|}{ Sex } \\
\hline Female & 1.00 & & \\
\hline Male & 0.93 & $\begin{array}{l}0.90- \\
0.96\end{array}$ & $<0.001$ \\
\hline \multicolumn{4}{|l|}{ HIV } \\
\hline Negative & 1.00 & & \\
\hline Positive & 0.86 & $\begin{array}{l}0.79- \\
0.93\end{array}$ & $<0.001$ \\
\hline
\end{tabular}

Factors associated with false positive symptoms screening results.

Multivariate analysis was also conducted to determine the factors associated with false positive symptom screening results. The factors included male sex APR 0.96 (0.94-0.97) and positive HIV status APR $0.92(0.88-0.96)$ which are both associated with reduced false positive symptom results (Table 5).

Table 5

Multivariate analysis of the factors associated with a false positive Symptom result

\begin{tabular}{|llll|}
\hline Characteristic & Adjusted prevalence Ratio (APR) & 95\% Cl & P-value \\
\hline Sex & & & \\
Female & 1.00 & $0.88-0.96$ & $<0.001$ \\
Male & 0.92 & & \\
HIV & & & \\
Negative & 1.00 & $0.94-0.97$ & $<0.001$ \\
Positive & 0.96 & & \\
\hline
\end{tabular}

\section{Discussion}


This study is one of the first studies to compare the performance of the CXR or symptoms against culture confirmed pulmonary tuberculosis by LJ Method in a community tuberculosis screening setting such as the National Tuberculosis prevalence surveys.

Our results show that the CXR performs better than the symptoms in correlating with positive tuberculosis sputum culture results in community settings which agrees with other studies. ${ }^{(3,12,18-21)}$ The positive and negative likelihood ratios of the CXR versus symptoms further indicate that the CXR performs better in correlating tuberculosis sputum culture results. The results indicate that the use of the CXR in tuberculosis screening in community settings may hence increase tuberculosis case detection rate in high tuberculosis prevalence settings. This may be due to the increased number of individuals that are taken for the confirmatory tests. This is especially important in the era of the new advanced TB diagnostic tools.

Our results indicate that individuals above 45 years are more likely to have a false positive CXR when screened for tuberculosis. This may be explained by the increase in respiratory pathology associated with increase in age including pneumonia, chronic obstructive pulmonary disease among others. ${ }^{(22)}$ Therefore, the CXR is not a preferred TB screening test among these individuals.

HIV positive individuals and males are more likely to have a true diagnosis of tuberculosis with both the CXR and symptoms. (18) This is important in the diagnosis of tuberculosis since these populations have been shown to be at high risk for tuberculosis. ${ }^{(23)}$ However, other studies have shown that PLHIV have been found to have a lot of abnormalities on CXR and hence the CXR not a good TB diagnostic tool among such populations. ${ }^{(13)}$ Therefore further studies are required to investigate the discrepancy noted between our study and other studies.

The strengths of our study include community screening for tuberculosis as shown by the use of data collected during the UTPS. The study was reported according to the STARD (Standards for reporting of Diagnostics Accuracy Studies) 2015 guidelines. $^{(17)}$

Our study is subject to limitations. The fact that sputum culture was only done for participants with abnormal CXR or tuberculosis symptoms could have led to selection bias hence over estimation of the sensitivity of the CXR. However, the large sample size improves our estimation of the performance of the CXR.

We only looked at culture confirmed pulmonary tuberculosis but not clinically diagnosed and not culture negative or Extra Pulmonary TB. This could greatly impact the performance of symptoms screening and/ or CXR to identify true TB.

The study was performed under community screening conditions and therefore the results may not be applicable to tuberculosis screening in the hospital setting.

This study shows that community tuberculosis screening using the CXR improves tuberculosis case detection. Therefore, the CXR should be considered in community tuberculosis screening programmes.

\section{Conclusion}

This study made use of data obtained during the Uganda National Tuberculosis prevalence survey to determine the performance of the CXR in correlating tuberculosis culture results and factors associated with a false negative tuberculosis screening result. The results indicate that the CXR is a good tuberculosis screening tool and may improve TB case detection when used in community tuberculosis screening on addition to symptoms assessment. Individuals with knowledge to observe TB defining pathology on the CXR are adequate to provide expertise for CXR interpretation.

The use of the CXR in screening tuberculosis may improve TB case detection among the PLHIV and the males who are important risk groups for tuberculosis. However, the elderly patients above 45 years are likely to have false positive CXR results. Additional studies are needed to investigate the impact of the CXR screening tool in the era of the molecular 
diagnostic tests. The cost effectiveness of screening tuberculosis with the CXR in high TB prevalence settings also needs to be determined.

\section{List Of Abbreviations}

HIV Human Immune Virus

PLHIV People Living with HIV

IC Infection Control

ICF Intensified Case Finding

IPT Isoniazid Preventive Therapy

$\mathrm{MoH}$ Ministry of Health

NTLP National Tuberculosis and Leprosy Programme

PTB Pulmonary tuberculosis

SOMREC School of Medicine Research and Ethics Committee.

TB Tuberculosis

UTPS Uganda Tuberculosis Prevalence Survey

WHO World Health Organisation

\section{Declarations}

\section{Ethics approval and consent to participate}

Ethical approval of the original survey was granted by the institutional review boards of the Higher Degree Research and Ethics Committee at the Makerere University School of Public Health and the Uganda National Council of Science and Technology (reference IRB00011353).

The nested study obtained a waiver of Informed consent from the Makerere University School of Medicine research and Ethics Committee (SOMREC).

Participants in the primary study offered written informed consent and or assent. Permission to use the data was obtained from the Uganda NTLP, Ministry of Health.

\section{Consent for publication}

Not applicable

\section{Availability of data and materials}

All data generated or analysed during this study are included in this published article [and its supplementary information files]. 


\section{Competing interests}

The authors declare that they have no competing interests

\section{Funding}

The MakCHS - UCBerkeley- Yale Pulmonary Complications of AIDS Research Training (PART) Program, NIH D43TW009607, from the Fogarty International Center for the PART fellowship awarded to JN under which this work was completed. The funders had no role in study design, data collection and analysis, decision to publish, or preparation of the manuscript.

\section{Authors' contributions}

JN, IN, PL, FO, EK and JM contributed to conception, design, analysis, methodology, interpretation of data, drafting and revision.

FM contributed to conception, funding acquisition, data acquisition and writing.

RS contributed to the data curation, software acquisition and revision.

AK contributed to conception, funding acquisition, investigation, resources, supervision, validation, drafting and revision.

JBK contributed to conception, funding acquisition, investigation, supervision, validation, drafting and revision.

All authors read and approved the final manuscript.

\section{Acknowledgements}

The authors acknowledge the Ministry of Health Uganda through the National Tuberculosis and Leprosy Program for permission to utilise the data from the National Tuberculosis Survey 2014- 2015. The members of the department of clinical epidemiology and biostatistics of Makerere University College of health sciences school of medicine for their assistance in drafting the proposal. The MakCHS - UCBerkeley-Yale Pulmonary Complications of AIDS Research Training (PART) Program, NIH D43TW009607, from the Fogarty International Center for the PART fellowship awarded to JN under which this work was completed. The funders had no role in study design, data collection and analysis, decision to publish, or preparation of the manuscript.

The authors declare no conflict of interest.

\section{References}

1. Organization WH. Global tuberculosis report 2019. Geneva (Switzerland): World Health Organization; 2019. 2019.

2. WHO. <WHO_HQ_Reports-G2-PROD-EXT-TBCountryProfile Uganda.pdf>. 2018.

3. WHO. Systematic screening for active tuberculosis: principles and recommendations: World Health Organization; 2013.

4. WHO. The End TB Strategy: global strategy and targets for tuberculosis prevention, care and control after 2015. Geneva, Switzerland: WHO, 2015. 2016.

5. Claassens M, Van Schalkwyk C, Floyd S, Ayles H, Beyers N. Symptom screening rules to identify active pulmonary tuberculosis: Findings from the Zambian South African Tuberculosis and HIV/AIDS Reduction (ZAMSTAR) trial prevalence surveys. PLoS One. 2017;12(3):e0172881. 
6. Van't Hoog A, Langendam M, Mitchell E, Cobelens F, Sinclair D, Leeflang M, et al. A systematic review of the sensitivity and specificity of symptom-and chest-radiography screening for active pulmonary tuberculosis in HIV-negative persons and persons with unknown HIV status. REPORT-Version March 2013. World Health Organization, Geneva, Switzerland: WHO. 2013.

7. Shah N, Anh M, Thuy T, Thom D, Linh T, Nghia D, et al. Population-based chest X-ray screening for pulmonary tuberculosis in people living with HIV/AIDS, An Giang, Vietnam. The International Journal of Tuberculosis and Lung Disease. 2008;12(4):404-10.

8. Aluoch JA, Swai OB, Edwards EA, Stott H, Darbyshire JH, Fox W, et al. Study of Case-Finding for Pulmonary Tuberculosis in Outpatients Complaining of a Chronic Cough at a District Hospital in Kenya 1. American Review of Respiratory Disease. 1984;129(6):915-20.

9. Jaeger S, Karargyris A, Candemir S, Folio L, Siegelman J, Callaghan F, et al. Automatic tuberculosis screening using chest radiographs. IEEE transactions on medical imaging. 2014;33(2):233-45.

10. Den Boon S, Bateman E, Enarson D, Borgdorff M, Verver S, Lombard C, et al. Development and evaluation of a new chest radiograph reading and recording system for epidemiological surveys of tuberculosis and lung disease. The International Journal of Tuberculosis and Lung Disease. 2005;9(10):1088-96.

11. Gupta RK, Lawn SD, Bekker L-G, Caldwell J, Kaplan R, Wood R. Impact of HIV and CD4 count on tuberculosis diagnosis: analysis of citywide data from Cape Town, South Africa. The international journal of tuberculosis and lung disease : the official journal of the International Union against Tuberculosis and Lung Disease. 2013;17(8):1014-22.

12. Shah S, Demissie M, Lambert L, Ahmed J, Leulseged S, Kebede T, et al. Intensified tuberculosis case finding among HIVInfected persons from a voluntary counseling and testing center in Addis Ababa, Ethiopia. JAIDS Journal of Acquired Immune Deficiency Syndromes. 2009;50(5):537-45.

13. Mosimaneotsile B, Talbot E, Moeti T, Hone N, Moalosi G, Moffat $\mathrm{H}$, et al. Value of chest radiography in a tuberculosis prevention programme for HIV-infected people, Botswana. The Lancet. 2003;362(9395):1551-2.

14. Pepper T, Joseph P, Mwenya C, McKee G, Haushalter A, Carter A, et al. Normal chest radiography in pulmonary tuberculosis: implications for obtaining respiratory specimen cultures. The international journal of tuberculosis and lung disease. 2008;12(4):397-403.

15. MoH. Uganda National TB prevalence Survey 2014- 2015 Uganda Ministry of Health Website Ministry of Health 2017 [cited 2019 16th March 2019]. Report ]. Available from: http://health.go.ug/content/uganda-national-tuberculosisprevalence-survey-2014-2015-survey-report.

16. Den Boon S, White N, Van Lill S, Borgdorff M, Verver S, Lombard C, et al. An evaluation of symptom and chest radiographic screening in tuberculosis prevalence surveys. The International Journal of Tuberculosis and Lung Disease. 2006;10(8):876-82.

17. Cohen JF, Korevaar DA, Altman DG, Bruns DE, Gatsonis CA, Hooft L, et al. STARD 2015 guidelines for reporting diagnostic accuracy studies: explanation and elaboration. BMJ open. 2016;6(11):e012799.

18. Nguyen D, Bang N, Hung N, Beasley R, Hwang L, Graviss E. Yield of chest radiograph in tuberculosis screening for HIVinfected persons at a district-level HIV clinic. The International Journal of Tuberculosis and Lung Disease.

2016;20(2):211-7.

19. Khan FA, Verkuijl S, Parrish A, Chikwava F, Ntumy R, El-Sadr W, et al. Performance of symptom-based tuberculosis screening among people living with HIV: not as great as hoped. AIDS. 2014;28(10):1463-72.

20. Story A, Aldridge R, Abubakar I, Stagg H, Lipman M, Watson J, et al. Active case finding for pulmonary tuberculosis using mobile digital chest radiography: an observational study. The International Journal of Tuberculosis and Lung Disease. 2012;16(11):1461-7.

21. Organization WH. Systematic screening for active tuberculosis: principles and recommendations: World Health Organization; 2013. 
22. Li J, Chung P-H, Leung CL, Nishikiori N, Chan EY, Yeoh E-K. The strategic framework of tuberculosis control and prevention in the elderly: a scoping review towards End TB targets. Infectious diseases of poverty. 2017;6(1):70.

23. Kirenga BJ, Ssengooba W, Muwonge C, Nakiyingi L, Kyaligonza S, Kasozi S, et al. Tuberculosis risk factors among tuberculosis patients in Kampala, Uganda: implications for tuberculosis control. BMC public health. 2015;15(1):1.

\section{Figures}

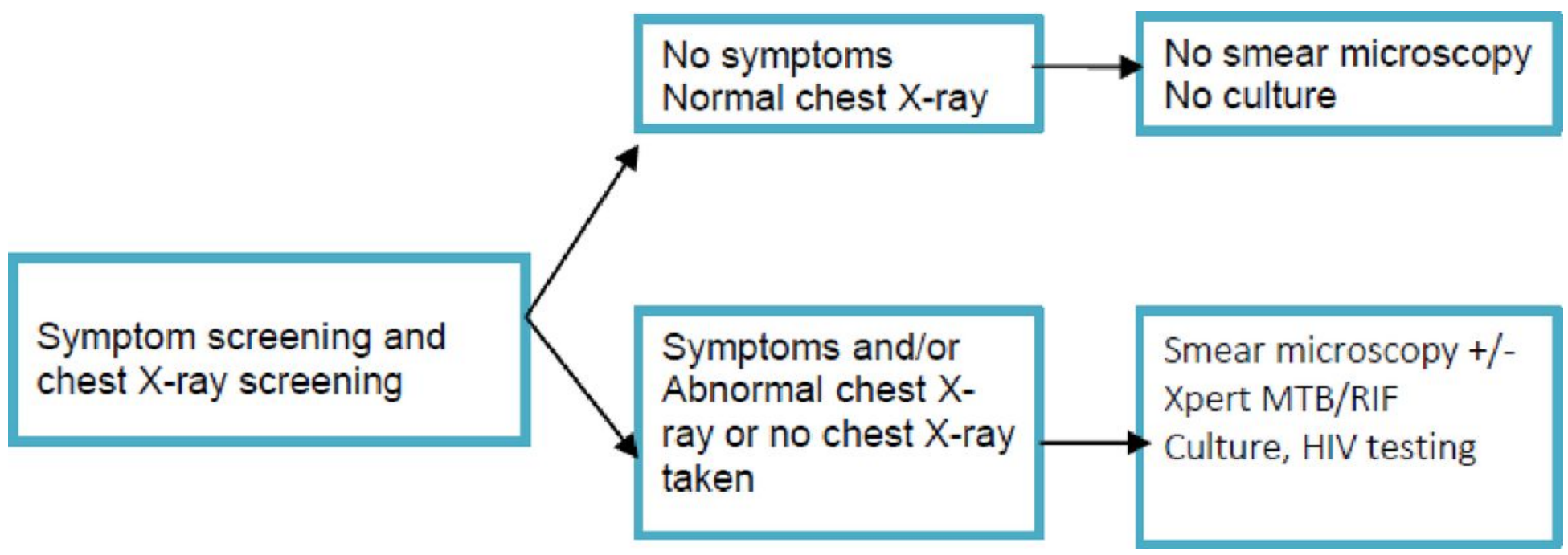

Figure 1

Diagrammatic representation of the combined symptom and chest x-ray screening strategy. 


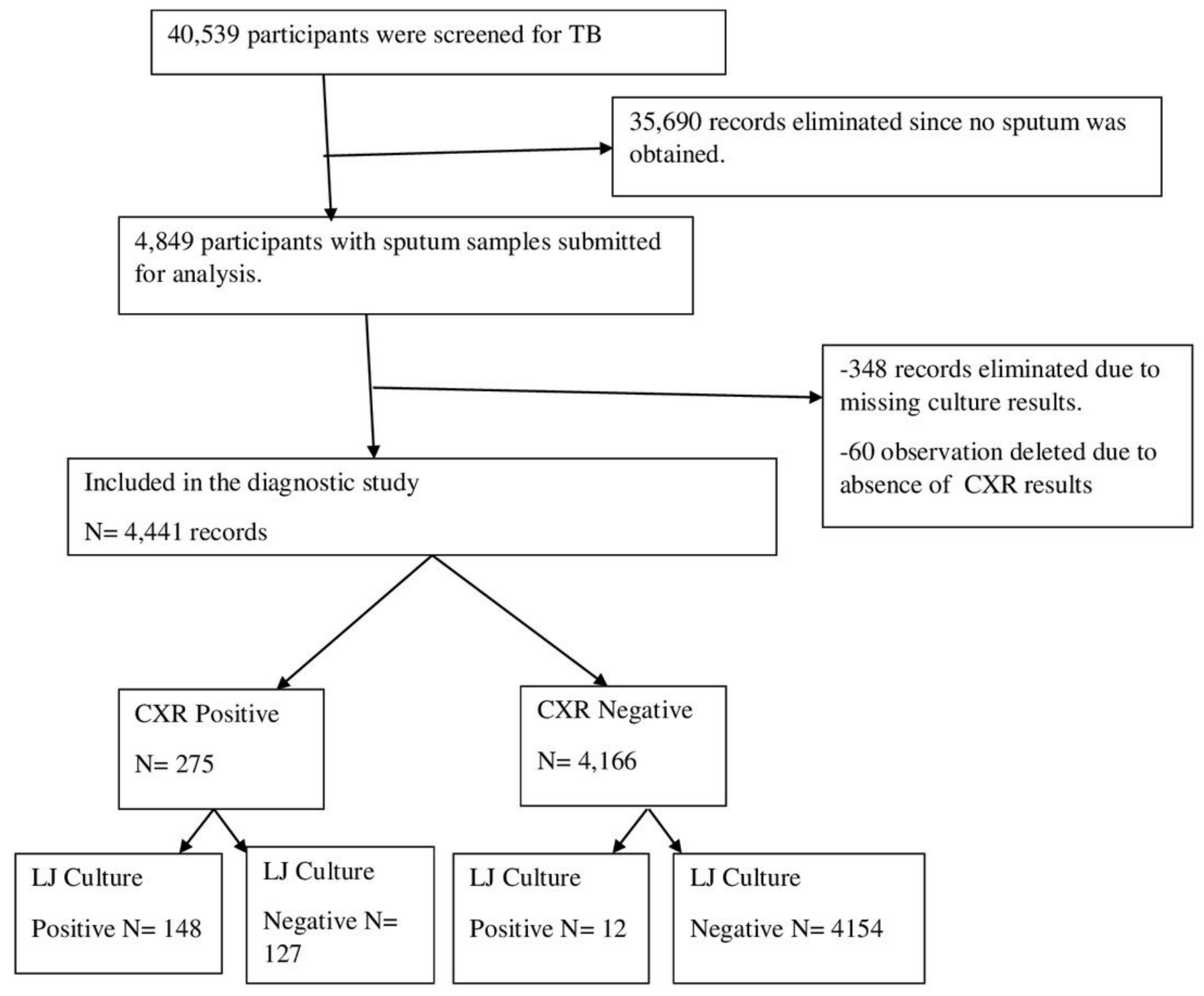

Figure 2

Study Profile 


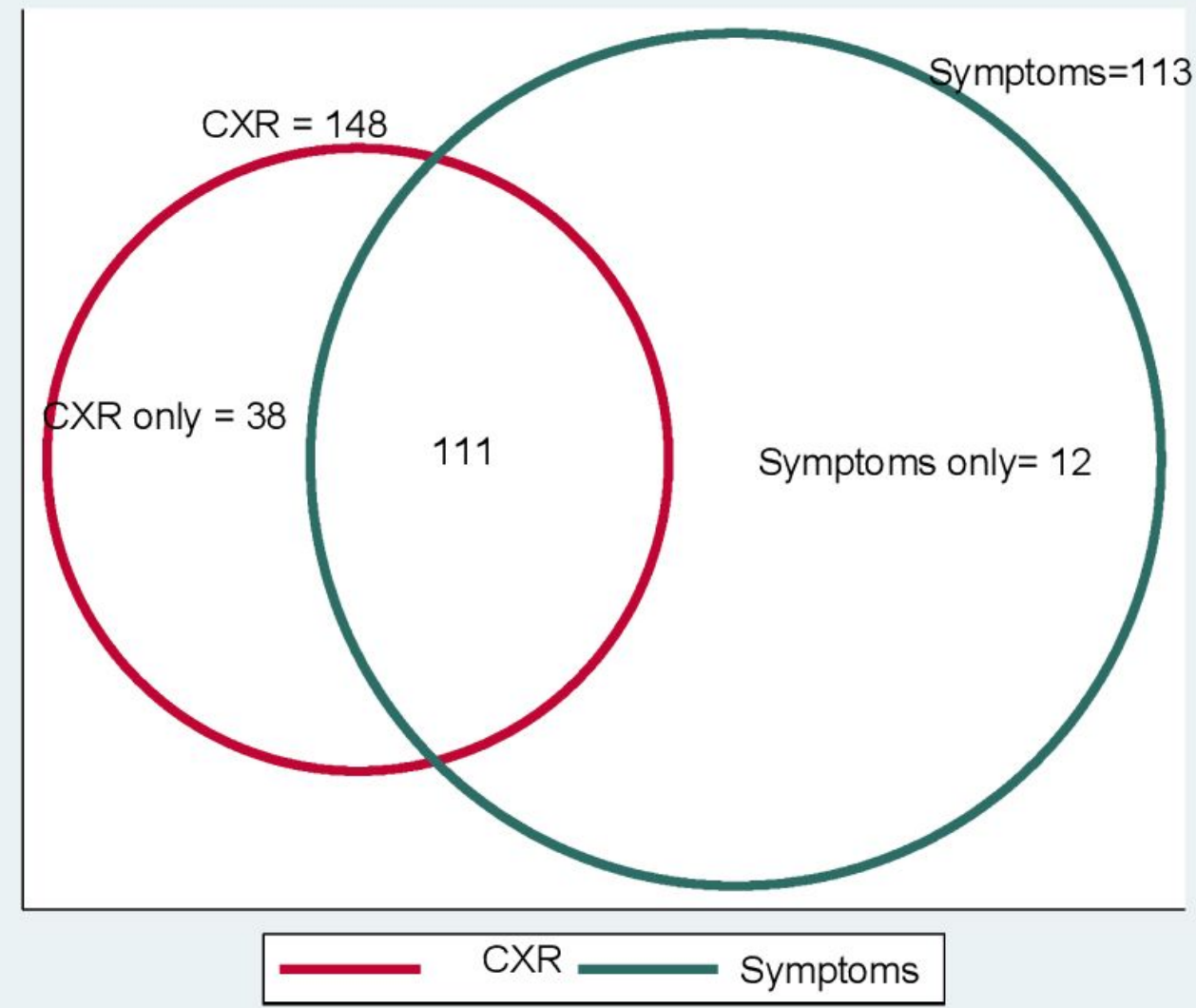

Figure 3

A Venn diagram of the culture confirmed Tuberculosis cases per screening tool.

\section{Supplementary Files}

This is a list of supplementary files associated with this preprint. Click to download.

- AdditionalFile1.xls 